\title{
Say goodbye to the cancer pain with a warming heart - the 2010 year's heart-warming party of Tongji Hospital in Wuhan
}

Author: Yi Cheng, from the Oncology Department of Tongji Hospital, Tongji Medical College, Huazhong University of Science and Technology, Wuhan 430030, China

Photo: Qiaoli Peng, Xi Yang, Yangyang Yu, from Tongji College, Huazhong University of Science and Technology, Wuhan 430030, China

On January 14th, 2010, the 2010 year's heart-warming party was held in the Tongji Cancer Center of Wuhan Tongji Hospital. This party was held by the the oncology department of Wuhan Tongji Hospital and the "sunshine medical students", the branch of Tongji medical school young volunteers association. Patients, medical staff and young volunteers gathered to celebrate the new year.

In this party, the patient enjoyed many wonderful shows, including Shaoxing opera, fluting and guitar. On this excellent party, a cancer patient, named Li Dengxi, played the harmonica, and then shared his experience of how to fight cancer and pain. He said :"Nothing is more important than your optimistic attitude when facing cancer and pain. If we obey the medical staff's advice and

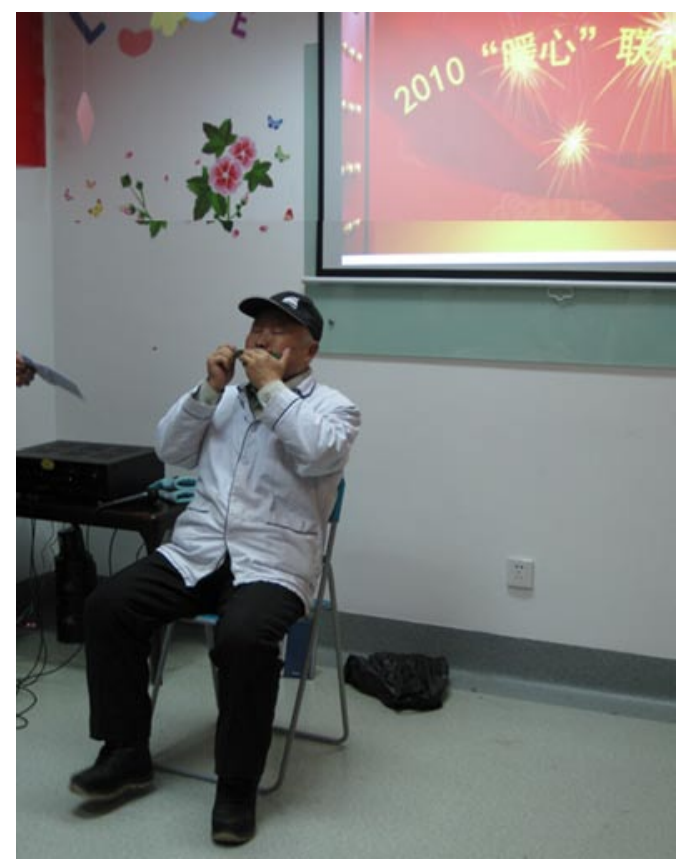

Li Dengxi was playing the harmonica

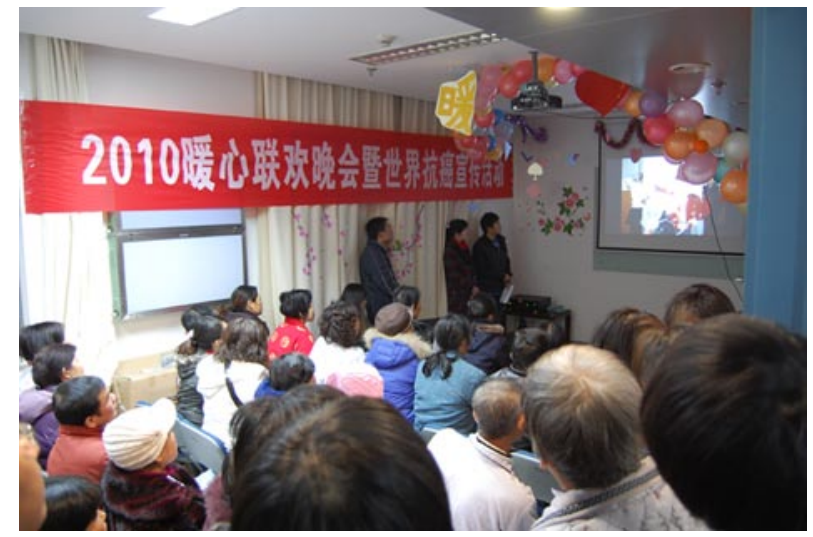

Activities on-site

keep ourselves in a peaceful mood, we can finally defeat the cancer and pain."

The highlight of the party was the Q\&A game. Every patient in the party got a manual, provided by Beijing Mundipharma Pharmaceutical Co., Ltd. The information in the manual referred to the common misunderstanding about the cancer pain and its treatment, which were also the context of the questions. What the patients could get is not only the presents in the game, but also the correct concept and sense, and thus the confidence to treat the pain.

The "sunshine medical students", the branch of Tongji medical school young volunteers association was founded in April, 2008. The members of the branch, together with the medical staff in the Tongji Cancer Center of $\mathrm{Wu}$ han Tongji Hosptial, served the cancer patients with psychological guidance and health education. Yu Xuan, the chairman of the branch, said: "We should develop the work together with the medical, to relieve the patients' physical and psychological pain, warm their heart and brighten their future." 